\title{
ON LOCAL BORG-MARCHENKO UNIQUENESS RESULTS
}

\author{
FRITZ GESZTESY ${ }^{1}$ AND BARRY SIMON ${ }^{2}$
}

\begin{abstract}
We provide a new short proof of the following fact, first proved by one of us in 1998: If two Weyl-Titchmarsh $m$ functions, $m_{j}(z)$, of two Schrödinger operators $H_{j}=-\frac{d^{2}}{d x^{2}}+q_{j}$, $j=1,2$ in $L^{2}((0, R)), 0<R \leq \infty$, are exponentially close, that is, $\left|m_{1}(z)-m_{2}(z)\right| \underset{|z| \rightarrow \infty}{=} O\left(e^{-2 \operatorname{Im}\left(z^{1 / 2}\right) a}\right), 0<a<R$, then $q_{1}=q_{2}$ a.e. on $[0, a]$. The result applies to any boundary conditions at $x=0$ and $x=R$ and should be considered a local version of the celebrated Borg-Marchenko uniqueness result (which is quickly recovered as a corollary to our proof). Moreover, we extend the local uniqueness result to matrix-valued Schrödinger operators.
\end{abstract}

\section{INTRODUCTION}

Let $H_{j}=-\frac{d^{2}}{d x^{2}}+q_{j}, q_{j} \in L^{1}([0, R])$ for all $R>0, q_{j}$ real-valued, $j=1,2$, be two self-adjoint operators in $L^{2}([0, \infty))$ with a Dirichlet boundary condition at $x=0_{+}$. Let $m_{j}(z), z \in \mathbb{C} \backslash \mathbb{R}$ be the WeylTitchmarsh $m$-functions associated with $H_{j}, j=1,2$. The principal purpose of this note is to provide a short proof of the following uniqueness theorem in the spectral theory of one-dimensional Schrödinger operators, originally obtained by Simon 33 in 1998. (Actually, Simon's result [33] was weaker; the result as stated is from [11].)

Theorem 1.1. Let $a>0,0<\varepsilon<\pi / 2$ and suppose that

$$
\left|m_{1}(z)-m_{2}(z)\right| \underset{|z| \rightarrow \infty}{=} O\left(e^{-2 \operatorname{Im}\left(z^{1 / 2}\right) a}\right)
$$

\footnotetext{
${ }^{1}$ Department of Mathematics, University of Missouri, Columbia, MO 65211, USA. E-mail: fritz@math.missouri.edu

${ }^{2}$ Division of Physics, Mathematics, and Astronomy, 253-37, California Institute of Technology, Pasadena, CA 91125, USA. E-mail: bsimon@caltech.edu. This material is based upon work supported by the National Science Foundation under Grant No. DMS-9707661. The Government has certain rights in this material.

Date: October 15, 1999.

1991 Mathematics Subject Classification. Primary: 34A55, 34B20; Secondary: 34L05, 47A10.

Key words and phrases. Inverse spectral theory, Weyl-Titchmarsh $m$-function, uniqueness theorems.
} 
along the ray $\arg (z)=\pi-\varepsilon$. Then

$$
q_{1}(x)=q_{2}(x) \text { for a.e. } x \in[0, a] .
$$

For reasons of brevity we stated Theorem 1.1 only in the simplest possible case. Extensions to finite intervals $[0, R]$ instead of the halfline $[0, \infty)$, a discussion of boundary conditions other than Dirichlet at $x=0_{+}$, and the case of matrix-valued Schrödinger operators - a new result — will be provided in the main body of this paper.

Theorem 1.1 should be viewed as a local (and hence stronger) version of the following celebrated Borg-Marchenko uniqueness theorem, published by Marchenko [25] in 1950. Marchenko's extensive treatise on spectral theory of one-dimensional Schrödinger operators [26], repeating the proof of his uniqueness theorem, then appeared in 1952, which also marked the appearance of Borg's proof of the uniqueness theorem [5] (apparently, based on his lecture at the 11th Scandinavian Congress of Mathematicians held at Trondheim, Norway in 1949).

Theorem 1.2. ([5, 25, 26]) Suppose

$$
m_{1}(z)=m_{2}(z), \quad z \in \mathbb{C} \backslash \mathbb{R},
$$

then

$$
q_{1}(x)=q_{2}(x) \text { for a.e. } x \in[0, \infty) .
$$

Again, we emphasize that Borg and Marchenko also treat the general case of non-Dirichlet boundary conditions at $x=0_{+}$, whose discussion we defer to Section 2. Moreover, Marchenko simultaneously discussed the half-line and finite interval case, also to be deferred to Section 2.

As pointed out by Levitan [23] in the Notes to Chapter 2, Borg and Marchenko were actually preceded by Tikhonov [34] in 1949, who proved a special case of Theorem 1.2 in connection with the string equation (and hence under certain additional hypotheses on $q_{j}$ ). Since WeylTitchmarsh functions $m(z)$ are uniquely related to the spectral measure $d \rho$ of a self-adjoint (Dirichlet) Schrödinger operator $H=-\frac{d^{2}}{d x^{2}}+q$ in $L^{2}([0, \infty))$ by the standard Herglotz representation

$$
m(z)=\operatorname{Re}(m(i))+\int_{\mathbb{R}} d \rho(\lambda)\left[(\lambda-z)^{-1}-\lambda\left(1+\lambda^{2}\right)^{-1}\right], \quad z \in \mathbb{C} \backslash \mathbb{R},
$$

Theorem 1.2 is equivalent to the following statement: Denote by $d \rho_{j}$ the spectral measures of $H_{j}, j=1,2$. Then

$$
d \rho_{1}=d \rho_{2} \text { implies } q_{1}=q_{2} \text { a.e. on }[0, \infty) \text {. }
$$

In fact, Marchenko's proof takes the spectral measures $d \rho_{j}$ as the point of departure while Borg focuses on the Weyl-Titchmarsh functions $m_{j}$. 
To the best of our knowledge, the only alternative approaches to Theorem 1.2 are based on the Gelfand-Levitan solution of the inverse spectral problem published in 1951 (see also Levitan and Gasymov [24]) and alternative variants due to M. Krein [20], [21]. In particular, it took over 45 years to improve on Theorem 1.2 and derive its local counterpart, Theorem 1.1. While the original proof of Theorem 1.1 in 33 relied on the full power of a new formalism in inverse spectral theory, relating $m(z)$ to finite Laplace transforms of the type

$$
m(z)=i z^{1 / 2}-\int_{0}^{a} d \alpha A(\alpha) e^{2 \alpha i z^{1 / 2}}+\widetilde{O}\left(e^{2 \alpha i z^{1 / 2}}\right)
$$

as $|z| \rightarrow \infty$ with $\arg (z) \in(\varepsilon, \pi-\varepsilon)$ for some $0<\varepsilon<\pi$ (with $f=\widetilde{O}(g)$ if $g \rightarrow 0$ and for all $\left.\delta>0,\left(\frac{f}{g}\right)|g|^{\delta} \rightarrow 0\right)$, we will present a short and fairly elementary argument in Section 2. In fact, as a corollary to our new proof of Theorem 1.1, we also obtain an elementary proof of a strengthened version of Theorem 1.2.

We should also mention some work of Ramm [30], [31], who provided a proof of Theorem 1.2 under a very strong additional assumption, namely, that $q_{1}$ and $q_{2}$ are both of short range. While his result is necessarily weaker than the original Borg-Marchenko result, Theorem 1.2, his method of proof has elements in common with parts of our proof (namely, he uses (2.28) below with $a=\infty$ and obtains a Volterra integral equation close to our (2.34)).

Finally, we have in preparation [12] still another alternate proof of the local Borg-Marchenko theorem.

Extensions to finite intervals and general (i.e., non-Dirichlet) boundary conditions complete Section 2. Matrix-valued extensions of Theorem 1.1 are presented in Section 3 .

\section{A New Proof of Theorem 1.1}

Throughout this section, unless explicitly stated otherwise, potentials $q$ are supposed to satisfy

$$
q \in L^{1}([0, R]) \text { for all } R>0, \quad q \text { real-valued. }
$$

Given $q$, we introduce the corresponding self-adjoint Schrödinger operator $H$ in $L^{2}([0, \infty))$ with a Dirichlet boundary condition at $x=0_{+}$, by

$$
\begin{aligned}
& H=-\frac{d^{2}}{d x^{2}}+q \\
& \operatorname{dom}(H)=\left\{g \in L^{2}([0, \infty)) \mid g, g^{\prime} \in \mathrm{AC}([0, R]) \text { for all } R>0\right. \\
& \left.\quad g\left(0_{+}\right)=0, \text { s.-a.b.c. at } \infty ;\left(-g^{\prime \prime}+q g\right) \in L^{2}([0, \infty))\right\}
\end{aligned}
$$


Here "s.-a. b.c." denotes a self-adjoint boundary condition at $\infty$ (which becomes relevant only if $q$ is in the limit circle case at $\infty$, but should be discarded otherwise, i.e., in the limit point case, where such a boundary condition is automatically satisfied). For example, an explicit form of such a boundary condition is

$$
\lim _{x \uparrow \infty} W\left(f\left(z_{0}\right), g\right)(x)=0,
$$

where $f\left(z_{0}, x\right)$ for some fixed $z_{0} \in \mathbb{C} \backslash \mathbb{R}$, satisfies

$$
f\left(z_{0}, \cdot\right) \in L^{2}([0 \infty)), \quad-f^{\prime \prime}\left(z_{0}, x\right)+\left[q(x)-z_{0}\right] f\left(z_{0}, x\right)=0
$$

and $W(f, g)(x)=f(x) g^{\prime}(x)-f^{\prime}(x) g(x)$ denotes the Wronskian of $f$ and $g$. Since these possible boundary conditions hardly play a role in the analysis to follow, we will not dwell on them any further. (Pertinent details can be found in [10] and the references therein.)

Next, let $\psi(z, x)$ be the unique (up to constant multiples) Weyl solution associated with $H$, that is,

$$
\begin{aligned}
& \psi(z, \cdot) \in L^{2}([0, \infty)), \quad z \in \mathbb{C} \backslash \mathbb{R}, \\
& \psi(z, x) \text { satisfies the s.-a. b.c. of } H \text { at } \infty \text { (if any), } \\
& -\psi^{\prime \prime}(z, x)+[q(x)-z] \psi(z, x)=0 .
\end{aligned}
$$

Then the Weyl-Titchmarsh function $m(z)$ associated with $H$ is defined by

$$
m(z)=\psi^{\prime}\left(z, 0_{+}\right) / \psi\left(z, 0_{+}\right), \quad z \in \mathbb{C} \backslash \mathbb{R}
$$

and for later purposes we also introduce the corresponding $x$-dependent version, $m(z, x)$, by

$$
m(z, x)=\psi^{\prime}(z, x) / \psi(z, x), \quad z \in \mathbb{C} \backslash \mathbb{R}, \quad x \geq 0 .
$$

After these preliminaries we are now ready to state the main ingredients used in our new proof of Theorem 1.1.

Theorem 2.1. ([2, [1) Let $\arg (z) \in(\varepsilon, \pi-\varepsilon)$ for some $0<\varepsilon<\pi$. Then for any fixed $x \in[0, \infty)$,

$$
m(z, x) \underset{|z| \rightarrow \infty}{=} i z^{1 / 2}+o(1) .
$$

The following result shows that one can also get an estimate uniform in $x$ as long as $x$ varies in compact intervals.

Theorem 2.2. (匹11) Let $\arg (z) \in(\varepsilon, \pi-\varepsilon)$ for some $0<\varepsilon<\pi$, and suppose $\delta>0, a>0$. Then there exists a $C(\varepsilon, \delta, a)>0$ such that for all $x \in[0, a]$,

$$
\left|m(z, x)-i z^{1 / 2}\right| \leq C(\varepsilon, \delta, a)
$$


where $C(\varepsilon, \delta, a)$ depends on $\varepsilon, \delta$, and $\sup _{0 \leq x \leq a}\left(\int_{x}^{x+\delta} d y|q(y)|\right)$.

Theorems 2.1 and 2.2 can be proved following arguments of Atkinson [2], who studied the Riccati-type equation satisfied by $m(z, x)$,

$$
m^{\prime}(z, x)+m(z, x)^{2}=q(x)-z \text { for a.e. } x \geq 0 \text { and all } z \in \mathbb{C} \backslash \mathbb{R} \text {. }
$$

Next, let $q_{j}(x), j=1,2$ be two potentials satisfying (2.1), with $m_{j}(z)$ the associated (Dirichlet) $m$-functions. Combining the a priori bound (2.9) with the differential equation resulting from (2.10),

$$
\begin{aligned}
& {\left[m_{1}(z, x)-m_{2}(z, x)\right]^{\prime}} \\
& =q_{1}(x)-q_{2}(x)-\left[m_{1}(z, x)+m_{2}(z, x)\right]\left[m_{1}(z, x)-m_{2}(z, x)\right],
\end{aligned}
$$

permits one to prove the following converse of Theorem 1.1.

Theorem 2.3. (《11) Let $\arg (z) \in(\varepsilon, \pi-\varepsilon)$ for some $0<\varepsilon<\pi$ and suppose $a>0$. If

$$
q_{1}(x)=q_{2}(x) \text { for a.e. } x \in[0, a],
$$

then

$$
\left|m_{1}(z)-m_{2}(z)\right| \underset{|z| \rightarrow \infty}{=} O\left(e^{-2 \operatorname{Im}\left(z^{1 / 2}\right) a}\right) .
$$

Lemma 2.4. In addition to the hypotheses of Theorem 2.9, (resp., Theorem 2.3), suppose that $H$ (resp., $\left.H_{j}, j=1,2\right)$ is bounded from below. Then (2.9) (resp., (2.13)) extends to all $\arg (z) \in(\varepsilon, \pi]$.

Proof. Since $H_{x} \geq H$, where $H_{x}$ denotes the Schrödinger operator $-\frac{d^{2}}{d x^{2}}+q$ in $L^{2}([x, \infty))$ with a Dirichlet boundary condition at $x_{+}$ (and the same s.-a.b.c. at $\infty$ as $H$, if any), there is an $E_{0} \in \mathbb{R}$ such that for all $x \in[0, a], m(z, x)$ is analytic in $\mathbb{C} \backslash\left[E_{0}, \infty\right)$. Using $\overline{m(z, x)}=m(\bar{z}, x)$, the estimate (2.9) holds on the boundary of a sector with vertex at $E_{0}-1$, symmetry axis $\left(-\infty, E_{0}-1\right]$, and some opening angle $0<\varepsilon<\pi / 2$. An application of the Phragmén-Lindelöf principle (cf. [29, Part III, Sect. 6.5]) then extends (2.9) to all of the interior of that sector and hence in particular along the ray $z \downarrow-\infty$. Since (2.13) results from (2.9) upon integrating (cf. (2.11)),

$$
\begin{aligned}
& \left.m_{1}(z, x)-m_{2}(z, x)\right]^{\prime} \\
& =-\left[m_{1}(z, x)+m_{2}(z, x)\right]\left[m_{1}(z, x),-m_{2}(z, x)\right], \quad x \in[0, a]
\end{aligned}
$$

from $x=0$ to $x=a$, the extension of $(2.9)$ to $z$ with $\arg (z) \in(\varepsilon, \pi]$ just proven, allows one to estimate

$$
\left|m_{1}(z, x)+m_{2}(z, x)\right| \underset{|z| \rightarrow \infty}{=} 2 i z^{1 / 2}+O(1), \quad \arg (z) \in(\varepsilon, \pi],
$$


uniformly with respect to $x \in[0, a]$, and hence to extend (2.13) to $\arg (z) \in(\varepsilon, \pi]$.

Next, we briefly recall a few well-known facts on compactly supported $q$. Hence we suppose temporarily that

$$
\sup (\operatorname{supp}(q))=\alpha<\infty .
$$

In this case, the Jost solution $f(z, x)$ associated with $q(x)$ satisfies

$$
\begin{aligned}
f(z, x) & =e^{i z^{1 / 2} x}-\int_{x}^{\alpha} d y \frac{\sin \left(z^{1 / 2}(x-y)\right)}{z^{1 / 2}} q(y) f(z, y) \\
& =e^{i z^{1 / 2} x}+\int_{x}^{\alpha} d y K(x, y) e^{i z^{1 / 2} y}, \quad \operatorname{Im}\left(z^{1 / 2}\right) \geq 0, x \geq 0
\end{aligned}
$$

where $K(x, y)$ denotes the transformation kernel satisfying (cf. 27, Sect. 3.1])

$$
\begin{aligned}
& K(x, y)=\frac{1}{2} \int_{(x+y) / 2}^{\alpha} d x^{\prime} q\left(x^{\prime}\right)-\int_{(x+y) / 2}^{\alpha} \int_{0}^{(y-x) / 2} d x^{\prime \prime} q\left(x^{\prime}-x^{\prime \prime}\right) \times \\
& \times K\left(x^{\prime}-x^{\prime \prime}, x^{\prime}+x^{\prime \prime}\right), \quad x \leq y, \\
& K(x, y)=0, \quad x>y, \\
& \left|K(x, y) \leq \frac{1}{2} \int_{(x+y) / 2}^{\alpha} d x^{\prime}\right| q\left(x^{\prime}\right) \mid \exp \left(\int_{x}^{\alpha} d x^{\prime \prime} x^{\prime \prime}\left|q\left(x^{\prime \prime}\right)\right|\right) .
\end{aligned}
$$

Moreover, $f(z, x)$ is a multiple of the Weyl solution, implying

$$
m(z, x)=f^{\prime}(z, x) / f(z, x), \quad z \in \mathbb{C} \backslash \mathbb{R}, x \geq 0,
$$

and the Volterra integral equation (2.17) immediately yields

$$
\begin{gathered}
|f(z, x)| \leq C e^{-\operatorname{Im}\left(z^{1 / 2}\right) x}, \quad \operatorname{Im}\left(z^{1 / 2}\right) \geq 0, x \geq 0, \\
f(z, x) \underset{\substack{|z| \rightarrow \infty \\
\operatorname{Im}\left(z^{1 / 2}\right) \geq 0}}{=} e^{i z^{1 / 2} x}\left(1+O\left(|z|^{-1 / 2}\right), \quad x \geq 0,\right.
\end{gathered}
$$

Our final ingredient concerns the following result on finite Laplace transforms.

Lemma 2.5. (= Lemma A.2.1 in [33]) Let $g \in L^{1}([0, a])$ and assume that $\int_{0}^{a} d y g(y) e^{-x y} \underset{x \uparrow \infty}{=} O\left(e^{-x a}\right)$. Then $g(y)=0$ for a.e. $y \in[0, a]$.

Given these facts, the proof of Theorem 1.1 now becomes quite simple. 
Proof of Theorem 1.1. By Theorem 2.3 we may assume, without loss of generality, that $q_{1}$ and $q_{2}$ are compactly supported such that

$$
\operatorname{supp}\left(q_{j}\right) \subseteq[0, a], \quad j=1,2,
$$

and by Lemma 2.4 we may suppose that (1.1) holds along the ray $z \downarrow-\infty$, that is,

$$
\left|m_{1}(z)-m_{2}(z)\right|_{z \downarrow-\infty}^{=} O\left(e^{-2|z|^{1 / 2} a}\right) .
$$

Denoting by $m_{j}(z, x)$ and $f_{j}(z, x)$ the $m$-functions and Jost solutions associated with $q_{j}, j=1,2$, integrating the elementary identity

$$
\frac{d}{d x} W\left(f_{1}(z, x), f_{2}(z, x)\right)=-\left[q_{1}(x)-q_{2}(x)\right] f_{1}(z, x) f_{2}(z, x)
$$

from $x=0$ to $x=a$, taking into account (2.22), yields

$$
\begin{aligned}
& \int_{0}^{a} d x\left[q_{1}(x)-q_{2}(x)\right] f_{1}(z, x) f_{2}(z, x) \\
& =\left.f_{1}(z, x) f_{2}(z, x)\left[m_{1}(z, x)-m_{2}(z, x)\right]\right|_{x=0} ^{a} .
\end{aligned}
$$

By (2.8), (2.23), and (2.26), the right-hand side of (2.28) is $O\left(e^{-2|z|^{1 / 2} a}\right)$ as $z \downarrow-\infty$, that is,

$$
\int_{0}^{a} d x\left[q_{1}(x)-q_{2}(x)\right] f_{1}(z, x) f_{2}(z, x) \underset{z \downarrow-\infty}{=} O\left(e^{-2|z|^{1 / 2} a}\right) .
$$

Denoting by $K_{j}(x, y)$ the transformation kernels associated with $q_{j}$, $j=1,2$, 2.18) implies

$$
f_{1}(z, x) f_{2}(z, x)=e^{2 i z^{1 / 2} x}+\int_{x}^{a} d y L(x, y) e^{2 i z^{1 / 2} y}
$$

where

$$
\begin{aligned}
L(x, y)= & 2\left[K_{1}(x, 2 y-x)+K_{2}(x, 2 y-x)\right] \\
& +2 \int_{x}^{2 y-x} d x^{\prime} K_{1}\left(x, x^{\prime}\right) K_{2}\left(x, 2 y-x^{\prime}\right), \quad x \leq y, \\
L(x, y)= & 0, \quad x>y \quad \text { or } \quad y>a .
\end{aligned}
$$

Insertion of (2.30) into (2.29), interchanging the order of integration in the double integral, then yields

$$
\begin{aligned}
& \int_{0}^{a} d x\left[q_{1}(x)-q_{2}(x)\right] f_{1}(z, x) f_{2}(z, x) \\
& =\int_{0}^{a} d y\left\{\left[q_{1}(y)-q_{2}(y)\right]+\int_{0}^{y} d x L(x, y)\left[q_{1}(x)-q_{2}(x)\right]\right\} e^{-2|z|^{1 / 2} y}
\end{aligned}
$$




$$
\underset{z \downarrow-\infty}{=} O\left(e^{-2|z|^{1 / 2} a}\right)
$$

An application of Lemma 2.5 then yields

$$
\left[q_{1}(y)-q_{2}(y)\right]+\int_{0}^{y} d x L(x, y)\left[q_{1}(x)-q_{2}(x)\right]=0 \quad \text { for a.e. } y \in[0 . a] \text {. }
$$

Since 2.34) is a homogeneous Volterra integral equation with a continuous integral kernel $L(x, y)$, one concludes $q_{1}=q_{2}$ a.e. on $[0, a]$.

In particular, one obtains the following strengthened version of the original Borg-Marchenko uniqueness result, Theorem 1.2 .

Corollary 2.6. Let $0<\varepsilon<\pi / 2$ and suppose that for all $a>0$,

$$
\left|m_{1}(z)-m_{2}(z)\right| \underset{|z| \rightarrow \infty}{=} O\left(e^{-2 \operatorname{Im}\left(z^{1 / 2}\right) a}\right)
$$

along the ray $\arg (z)=\pi-\varepsilon$. Then

$$
q_{1}(x)=q_{2}(x) \text { for a.e. } x \in[0, \infty) .
$$

Remark 2.7. The Borg-Marchenko uniqueness result, Theorem 1.2 (but not our strengthened version, Corollary 2.6), under the additional condition of short-range potentials $q_{j}$ satisfying $q_{j} \in L^{1}([0, \infty) ;(1+x) d x)$, $j=1,2$, can also be proved using Property $\mathrm{C}$, a device recently used by Ramm 30, 31 in a variety of uniqueness results. In this case, (2.28) for $z=\lambda>0$ becomes

$$
\begin{aligned}
& \int_{0}^{\infty} d x\left[q_{1}(x)-q_{2}(x)\right] f_{1}(\lambda, x) f_{2}(\lambda, z) \\
& =-f_{1}(\lambda, 0) f_{2}(\lambda, 0)\left[m_{1}(\lambda+i 0)-m_{2}(\lambda+i 0)\right]=0, \quad \lambda>0
\end{aligned}
$$

since $m_{1}(z)=m_{2}(z), z \in \mathbb{C}_{+}$extends to $m_{1}(\lambda+i 0)=m_{2}(\lambda+i 0)$, $\lambda>0$ by continuity in the present short-range case. By definition, Property $\mathrm{C}$ stands for completeness of the set $\left\{f_{1}(\lambda, x) f_{2}(\lambda, x)\right\}_{\lambda>0}$ in $L^{1}([0, \infty) ;(1+x) d x)$ (this extends to $L^{1}([0, \infty))$ ) and hence (2.37) yields $q_{1}=q_{2}$ a.e. on $[0, \infty)$.

In the remainder of this section, we consider a variety of generalizations of the result obtained.

Remark 2.8. The ray $\arg (z)=\pi-\varepsilon, 0<\varepsilon<\pi / 2$ chosen in Theorem 1.1 and Corollary 2.6 is of no particular importance. A limit taken along any non-self-intersecting curve $\mathcal{C}$ going to infinity in the sector $\arg (z) \in(\pi / 2+\varepsilon, \pi-\varepsilon)$ will do as we can apply the Phragmén-Lindelöf principle (29, Part III, Sect. 6.5]) to the region enclosed by $\mathcal{C}$ and its 
complex conjugate $\overline{\mathcal{C}}$ (needed in connection with Lemma 2.4 in order to reduce the general case to the case of spectra bounded from below).

Remark 2.9. For simplicity of exposition, we only discussed the Dirichlet boundary condition

$$
g\left(0_{+}\right)=0
$$

in the definition of $H$ in (2.2). Next we replace (2.38) by the general boundary condition

$$
\sin (\alpha) g^{\prime}\left(0_{+}\right)+\cos (\alpha) g\left(0_{+}\right)=0, \quad \alpha \in[0, \pi)
$$

in (2.2), denoting the resulting Schrödinger operator by $H_{\alpha}$, while keeping the boundary condition at infinity (if any) identical for all $\alpha \in[0, \pi)$. Denoting by $m_{\alpha}(z)$ the Weyl-Titchmarsh function associated with $H_{\alpha}$, the well-known relation (cf. e.g., Appendix A of [10 for precise details on $H_{\alpha}$ and $\left.m_{\alpha}(z)\right)$

$$
m_{\alpha}(z)=\frac{-\sin (\alpha)+\cos (\alpha) m(z)}{\cos (\alpha)+\sin (\alpha) m(z)}, \quad \alpha \in[0, \pi), z \in \mathbb{C} \backslash \mathbb{R}
$$

reduces the case $\alpha \in(0, \pi)$ to the Dirichlet case $\alpha=0$. In particular, Theorem 1.1 and Corollary 2.6 remain valid with $m_{j}(z)$ replaced by $m_{j, \alpha}(z), \alpha \in[0, \pi)$. Indeed, $\left|m_{1, \alpha}(z)-m_{2, \alpha}(z)\right| \underset{|z| \rightarrow \infty}{=} O\left(e^{-2 \operatorname{Im}\left(z^{1 / 2}\right) a}\right)$ along the $\operatorname{ray} \arg (z)=\pi-\varepsilon$ is easily seen to imply, for all sufficiently small $\delta>0$,

$$
\begin{aligned}
& \left|m_{1,0}(z)-m_{2,0}(z)\right| \underset{|z| \rightarrow \infty}{=} O\left(|z| e^{-2 \operatorname{Im}\left(z^{1 / 2}\right) a}\right) \\
& \underset{|z| \rightarrow \infty}{=} O\left(e^{-2 \operatorname{Im}\left(z^{1 / 2}\right)(a-\delta)}\right)
\end{aligned}
$$

along the ray $\arg (z)=\pi-\varepsilon$. Hence one infers from Theorem 1.1 that for all $0<\delta<a, q_{1}=q_{2}$ a.e. on $[0, a-\delta]$. Since $\delta>0$ can be chosen arbitrarily small, one concludes $q_{1}=q_{2}$ a.e. on $[0, a]$. In fact, more is true. Since $m_{\alpha}(z) \underset{|z| \rightarrow \infty}{\longrightarrow} \cot (\alpha)$ along the ray, one concludes that $\left|m_{1, \alpha_{1}}-m_{2, \alpha_{2}}(z)\right| \underset{|z| \rightarrow \infty}{=} O\left(e^{-2 \operatorname{Im}\left(z^{1 / 2}\right) a}\right)$ along a ray implies $\alpha_{1}=\alpha_{2}$ and $q_{1}=q_{2}$ a.e. on $[0, a]$.

Remark 2.10. If one is interested in a finite interval $[0, b]$ instead of the half-line $[0, \infty)$ in Theorem 1.1, with $0<a<b$, one introduces a self-adjoint boundary condition at $x=b_{-}$of the type

$$
\sin (\beta) g^{\prime}\left(b_{-}\right)+\cos (\beta) g\left(b_{-}\right)=0, \quad \beta \in[0, \pi) .
$$


The analog of the Weyl solution $\psi(z, x ; b, \beta)$ for the corresponding Schrödinger operator $H(b, \beta)$ in $L^{2}([0, b])$ defined by

$$
\begin{aligned}
& H(b, \beta)=-\frac{d^{2}}{d x^{2}}+q, \quad \beta \in[0, \pi), \\
& \operatorname{dom}(H(b, \beta))=\left\{g \in L^{2}([0, b]) \mid g, g^{\prime} \in \mathrm{AC}([0, b]) ; g\left(0_{+}\right)=0\right. \\
& \left.\quad \sin (\beta) g^{\prime}\left(b_{-}\right)+\cos (\beta) g\left(b_{-}\right)=0 ; \quad\left(-g^{\prime \prime}+q g\right) \in L^{2}([0, b])\right\}
\end{aligned}
$$

is then defined by

$$
\begin{aligned}
& \sin (\beta) \psi^{\prime}\left(z, b_{-} ; b, \beta\right)+\cos (\beta) \psi\left(z, b_{-} ; b, \beta\right)=0, \quad z \in \mathbb{C} \backslash \mathbb{R} \\
& -\psi^{\prime \prime}(z, x ; b, \beta)+[q(x)-z] \psi(z, x ; b, \beta)=0 .
\end{aligned}
$$

Moreover, the analog of (2.18) is then of the type

$$
\begin{array}{r}
\psi(z, x ; b, \beta)=\psi^{(0)}(z, x ; b, \beta)+\int_{x}^{b} d y K(x, y ; b, \beta) \psi^{(0)}(z, x ; b, \beta), \\
z \in \mathbb{C} \backslash \mathbb{R}, x \in[0, b],
\end{array}
$$

where

$$
\begin{aligned}
& \psi^{(0)}(z, x ; b, \beta)=\frac{e^{i z^{1 / 2} x}+\zeta(\beta, z) e^{i z^{1 / 2}(2 b-x)}}{1+\zeta(\beta, z) e^{2 i z^{1 / 2} b}}, \\
& \zeta(\beta, z)=\frac{-i z^{1 / 2}-\cot (\beta)}{-i z^{1 / 2}+\cot (\beta)}, \quad \operatorname{Im}\left(z^{1 / 2}\right) \geq 0
\end{aligned}
$$

is the corresponding Weyl solution in the case $q(x)=0, x \in[0, b]$, and $K(x, y ; b, \beta)$ is a transformation kernel analogous to $K(x, t ; h)$ discussed in Sect. 1.3 of [27]. Theorem 1.1 then extends to triples $\left(q_{j}, b_{j}, \beta_{j}\right), j=$ 1,2 with $a<\min \left(b_{1}, b_{2}\right)$, replacing $f_{j}(z, x)$ in $(2.29)$ by $\psi\left(z, x ; b_{j}, \beta_{j}\right)$, $j=1,2$. More precisely, if $m\left(z ; b_{j}, \beta_{j}\right)$ denote the $m$-functions for $H\left(b_{j}, \beta_{j}\right), j=1,2$ with $a<\min \left(b_{1}, b_{2}\right)$ and

$$
\left|m\left(z ; b_{1}, \beta_{1}\right)-m\left(z ; b_{2}, \beta_{2}\right)\right| \underset{|z| \rightarrow \infty}{=} O\left(e^{-2 \operatorname{Im}\left(z^{1 / 2}\right) a}\right)
$$

along the $\operatorname{ray} \arg (z)=\pi-\varepsilon$, then $q_{1}=q_{2}$ a.e. on $[0, a]$. In fact, it was precisely this version of Theorem 1.1 which was originally proven by one of us 33 in 1998.

One can also derive additional results in the case $a=b_{1}=b_{2}$ (cf. Theorem 1.3 in 33 ). Indeed, $\zeta(\beta, z) \underset{|z| \rightarrow \infty}{=} 1+O\left(|z|^{-1 / 2}\right)$, so by (2.45) and (2.46),

$$
\begin{aligned}
& \psi(z, 0 ; a, \beta) \underset{|z| \rightarrow \infty}{=} 1+O\left(|z|^{-1 / 2}\right), \\
& \psi(z, a ; a, \beta) \underset{|z| \rightarrow \infty}{=} 2 e^{i z^{1 / 2} a}\left(1+O\left(|z|^{-1 / 2}\right)\right),
\end{aligned}
$$


which are analogous to (2.24). Thus, if $q_{1}=q_{2}$ on $[0, a]$ but $\beta_{1} \neq \beta_{2}$, we have that

$$
\begin{aligned}
& {\left[m_{1}\left(z ; a, \beta_{1}\right)-m_{2}\left(z ; a, \beta_{2}\right)\right]} \\
& =\psi_{1}\left(z, 0 ; a, \beta_{1}\right)^{-1} \psi_{2}\left(z, 0 ; a, \beta_{2}\right)^{-1} W\left(\psi_{2}\left(z, 0 ; a, \beta_{2}\right), \psi_{1}\left(z, 0 ; a, \beta_{1}\right)\right) \\
& =\psi_{1}\left(z ; 0, a, \beta_{1}\right)^{-1} \psi_{2}\left(z, 0 ; a, \beta_{2}\right)^{-1} W\left(\psi_{2}\left(z, a ; a, \beta_{2}\right), \psi_{1}\left(z, a ; a, \beta_{2}\right)\right),
\end{aligned}
$$

by the constancy of the Wronskian when $q_{1}=q_{2}$. But $\psi^{\prime}(a) / \psi(a)$ equals $\cot (\beta)$ by $(2.44)$ and hence

$$
\begin{aligned}
m_{1}\left(z ; a, \beta_{1}\right)- & m_{2}\left(z ; a, \beta_{2}\right)=\psi_{1}\left(z, 0 ; a, \beta_{1}\right)^{-1} \psi_{2}\left(z, 0 ; a, \beta_{2}\right)^{-1} \times \\
& \times \psi_{1}\left(z, a ; a, \beta_{1}\right) \psi_{2}\left(z, a ; a, \beta_{2}\right)\left[\cot \left(\beta_{1}\right)-\cot \left(\beta_{2}\right)\right] .
\end{aligned}
$$

Using (2.48), (2.49), this implies that

$$
\begin{aligned}
& m_{1}\left(z ; a, \beta_{1}\right)-m_{2}\left(z ; a, \beta_{2}\right) \\
& =4 e^{2 i z^{1 / 2} a}\left[\cot \left(\beta_{1}\right)-\cot \left(\beta_{2}\right)\right]\left[1+O\left(|z|^{-1 / 2}\right)\right],
\end{aligned}
$$

which is Theorem 1.3 in 33 .

While we have separately described a few extensions in Remarks 2.82.10, it is clear that they can all be combined at once.

We also mention the analog of Theorem 1.1 for Schrödinger operators on the real line. Assuming

$$
q \in L_{\text {loc }}^{1}(\mathbb{R}), \quad q \text { real-valued, }
$$

one introduces the corresponding self-adjoint Schrödinger operator $H$ in $L^{2}(\mathbb{R})$ by

$$
\begin{aligned}
& H=-\frac{d^{2}}{d x^{2}}+q \\
& \operatorname{dom}(H)=\left\{g \in L^{2}(\mathbb{R}) \mid g, g^{\prime} \in \mathrm{AC}_{\text {loc }}(\mathbb{R}) ; \text { s. s.-a. b.c. at } \pm \infty ;\right. \\
& \left.\left(-g^{\prime \prime}+q g\right) \in L^{2}(\mathbb{R})\right\} .
\end{aligned}
$$

Here "s. s.-a. b.c." denotes separated self-adjoint boundary conditions at $+\infty$ and/or $-\infty$ (if any).

The $2 \times 2$ matrix-valued $m$-function $\mathcal{M}(z)$ associated with $H$ in $L^{2}(\mathbb{R})$ is then defined by

$$
\begin{aligned}
\mathcal{M}(z)= & \left(m_{-}(z)-m_{+}(z)\right)^{-1} \times \\
& \times\left(\begin{array}{cc}
1 & \left(m_{-}(z)+m_{+}(z)\right) / 2 \\
\left(m_{-}(z)+m_{+}(z)\right) / 2 & m_{-}(z) m_{+}(z)
\end{array}\right), \quad z \in \mathbb{C} \backslash \mathbb{R},
\end{aligned}
$$

where $m_{ \pm}(z)$ denote the half-line $m$-functions associated with $H$ restricted to $[0, \pm \infty)$ and a Dirichlet boundary condition at $x=0$. 
Next, let $q_{j}(x), j=1,2$ be two potentials satisfying (2.53) and $H_{j}$ the corresponding Schrödinger operators $(2.54)$ in $L^{2}(\mathbb{R})$, with $\mathcal{M}_{j}(z)$, $j=1,2$ the associated $2 \times 2$ matrix-valued $m$-functions. Then the analog of Theorem 1.1 reads as follows.

Theorem 2.11. Let $a>0,0<\varepsilon<\pi / 2$ and suppose that

$$
\left\|\mathcal{M}_{1}(z)-\mathcal{M}_{2}(z)\right\|_{\mathbb{C}^{2 \times 2}} \underset{|z| \rightarrow \infty}{=} O\left(e^{-2 \operatorname{Im}\left(z^{1 / 2}\right) a}\right)
$$

along the ray $\arg (z)=\pi-\varepsilon$. Then

$$
q_{1}(x)=q_{2}(x) \text { for a.e. } x \in[-a, a] .
$$

Proof. We denote by $m_{j, \pm}(z)$ the half-line (Dirichlet) $m$-functions associated with $H_{j}$ on $[0, \pm \infty), j=1,2$. Then a straightforward combination of (2.8) and (2.56) yields

$$
\left|m_{1, \pm}(z)-m_{2, \pm}(z)\right| \underset{|z| \rightarrow \infty}{=} O\left(|z| e^{-2 \operatorname{Im}\left(z^{1 / 2}\right) a}\right)
$$

and hence (2.57), applying Theorem 1.1 separately to the two half-lines $[0, \infty)$ and $(-\infty, 0]$ (and using the argument following (2.41).

Finally, the reader might be interested in the analog of Theorem 1.1 in the case of second-order difference operators, that is, Jacobi operators. Let $A$ be a bounded self-adjoint Jacobi operator in $\ell^{2}\left(\mathbb{N}_{0}\right)$ $\left(\mathbb{N}_{0}=\mathbb{N} \cup\{0\}\right)$ of the type

$$
A=\left(\begin{array}{cccccc}
b_{0} & a_{0} & 0 & 0 & \ldots & \ldots \\
a_{0} & b_{1} & a_{1} & 0 & \ldots & \ldots \\
0 & a_{1} & b_{2} & a_{2} & \ldots & \ldots \\
0 & 0 & a_{2} & \ddots & \ddots & \\
\vdots & \vdots & \vdots & \ddots & \ddots & \ddots \\
\vdots & \vdots & \vdots & & \ddots & \ddots
\end{array}\right), \quad a_{k}>0, b_{k} \in \mathbb{R}, k \in \mathbb{N}_{0}
$$

The corresponding $m$-function of $A$ is then defined by

$$
m(z)=\left(\delta_{0},(A-z)^{-1} \delta_{0}\right)=\int_{\mathbb{R}} d \rho(\lambda)(\lambda-z)^{-1}, \quad z \in \mathbb{C} \backslash \mathbb{R},
$$

where $\delta_{0}=(1,0,0, \ldots)$. The analog of Theorem 1.1 in the discrete case then reads as follows. Denote by $m_{j}(z)$ the $m$-functions for two self-adjoint Jacobi operators $A_{j}, j=1,2$, denoting the matrix elements of $A_{j}$ by $a_{j, k}, b_{j, k}, j=1,2, k \in \mathbb{N}_{0}$. Then

$$
\left|m_{1}(z)-m_{2}(z)\right| \underset{|z| \rightarrow \infty}{=} O\left(|z|^{-N}\right),
$$


for some $N \in \mathbb{N}, N \geq 3$, if and only if

$$
a_{1, k}=a_{2, k}, \quad b_{1, k}=b_{2, k}, \quad 0 \leq k \leq \frac{N-4}{2} \quad \text { if } N \text { is even } \quad(N \geq 4)
$$

and

$$
\begin{aligned}
& a_{1, k}=a_{2, k}, \quad 0 \leq k \leq \frac{N-5}{2} \\
& b_{1, k}=b_{2, k}, \quad 0 \leq k \leq \frac{N-3}{2} \quad \text { if } N \text { is odd } .
\end{aligned}
$$

The proof is clear from (2.60) and the well-known formulas (cf. \#, Sect. VII.1]).

$$
a_{k}=\int_{\mathbb{R}} d \rho(\lambda) \lambda P_{k}(\lambda) P_{k+1}(\lambda), \quad b_{k}=\int_{\mathbb{R}} d \rho(\lambda) \lambda P_{k}(\lambda)^{2}, \quad k \in \mathbb{N}_{0},
$$

where $\left\{P_{k}(\lambda)\right\}_{k \in \mathbb{N}_{0}}$ is an orthonormal system of polynomials with respect to the spectral measure $d \rho$, with $P_{k}(z)$ of degree $k$ in $z, P_{0}(z)=1$.

\section{Matrix-Valued Schrödinger Operators}

In our final section we extend Theorem 1.1 to matrix-valued potentials (cf., [6, Ch. III], [17], [22] and the references therein).

Let $m \in \mathbb{N}$ and denote by $I_{m}$ the identity matrix in $\mathbb{C}^{m}$. Assuming

$$
Q=Q^{*} \in L^{1}([0, R])^{m \times m} \text { for all } R>0,
$$

we introduce the corresponding matrix-valued self-adjoint Schrödinger operator $H$ in $L^{2}([0, \infty))^{m}$ with a Dirichlet boundary condition at $x=$ $0_{+}$, by

$$
\begin{aligned}
& H=-\frac{d^{2}}{d x^{2}} I_{m}+Q \\
& \operatorname{dom}(H)=\left\{g \in L^{2}([0, \infty))^{m} \mid g, g^{\prime} \in \mathrm{AC}([0, R])^{m} \text { for all } R>0\right. \\
& \left.\quad g\left(0_{+}\right)=0, \text { s.-a. b.c. at } \infty ;\left(-g^{\prime \prime}+Q g\right) \in L^{2}([0, \infty))^{m}\right\} .
\end{aligned}
$$

Here "s.-a. b.c. at $\infty$ " again denotes a self-adjoint boundary condition at $\infty$ (if $Q$ is not in the limit point case at $\infty$ ). For more details about the limit point/limit circle and all the intermediate cases, see 17, 13, 14, 15, 16, 18, 19, 28, 32 and the references therein.

Next, let $\Psi(z, x)$ be the unique (up to right multiplication of nonsingular constant $m \times m$ matrices) $m \times m$ matrix-valued Weyl solution associated with $H$, satisfying

$$
\Psi(z, \cdot) \in L^{2}([0, \infty))^{m \times m}, \quad z \in \mathbb{C} \backslash \mathbb{R}
$$




$$
\begin{aligned}
& \Psi(z, x) \text { satisfies the s.-a. b.c. of } H \text { at } \infty \text { (if any), } \\
& -\Psi^{\prime \prime}(z, x)+\left[Q(x)-z I_{m}\right] \Psi(z, x)=0 .
\end{aligned}
$$

The $m \times m$ matrix-valued Weyl-Titchmarsh function $M(z)$ associated with $H$ is then defined by

$$
M(z)=\Psi^{\prime}\left(z, 0_{+}\right) \Psi\left(z, 0_{+}\right)^{-1}, \quad z \in \mathbb{C} \backslash \mathbb{R}
$$

and similarly, we introduce its $x$-dependent version, $M(z, x)$, by

$$
M(z, x)=\Psi^{\prime}(z, x) \Psi(z, x)^{-1}, \quad z \in \mathbb{C} \backslash \mathbb{R}, x \geq 0 .
$$

The matrix Riccati equation satisfied by $M(z, x)$, the analog of (2.10), then reads

$$
M^{\prime}(z, x)+M(z, x)^{2}=Q(x)-z I_{m} \text { for a.e. } x \geq 0 \text { and all } z \in \mathbb{C} \backslash \mathbb{R} .
$$

Next, let $Q_{j}(x), j=1,2$ be two self-adjoint matrix-valued potentials satisfying (3.1), and $M_{j}(z), M_{j}(z, x)$ the Weyl-Titchmarsh matrices associated with the corresponding (Dirichlet) Schrödinger operators. Then the analog of (2.11) is of the form

$$
\begin{aligned}
& {\left[M_{1}(z, x)-M_{2}(z, x)\right]^{\prime}} \\
& =Q_{1}(x)-Q_{2}(x)-\frac{1}{2}\left[M_{1}(z, x)+M_{2}(z, x)\right]\left[M_{1}(z, x)-M_{2}(z, x)\right] \\
& \quad-\frac{1}{2}\left[M_{1}(z, x)-M_{2}(z, x)\right]\left[M_{1}(z, x)+M_{2}(z, x)\right] .
\end{aligned}
$$

Combining (3.9) with the elementary fact that any $m \times m$ matrix-valued solution $U(x)$ of

$$
U^{\prime}(x)=B(x) U(x)+U(x) B(x)
$$

is of the form

$$
U(x)=V(x) C W(x),
$$

where $C$ is a constant $m \times m$ matrix and $V(x)$, respectively, $W(x)$, is a fundamental system of solutions of $R^{\prime}(x)=B(x) R(x)$, respectively, $S^{\prime}(x)=S(x) B(x)$, one can prove the analogs of Theorems 2.1 2.3 in the present matrix context. More precisely, the matrix analogs of Theorems 2.1 and 2.2 follow from Theorem 4.8 in [7]. The corresponding analog of Theorem 2.3 follows from Theorem 4.5 and Remark 4.7 in [7]. Moreover, in the case that $H$ is bounded from below, Lemma 2.4 generalizes to the matrix-valued context and hence permits one to take the limit $z \downarrow-\infty$ in the matrix analog of (2.13). While the scalar case treated in detail in [11] is based on Riccati-type identities such as (2.11) and an a priori bound of the type (2.9) inspired by Atkinson's 1981 paper [2], the matrix-valued case discussed in depth in [7] is based 
on corresponding Riccati-type identities such as (3.9) and an a priori bound of the type

$$
M(z, x)=i z^{1 / 2} I_{m}+o\left(|z|^{1 / 2}\right)
$$

first obtained by Atkinson in an unpublished manuscript [3].

In the special case of short-range matrix-valued potentials $Q(x)$, $m \times m$ matrix analogs of the Jost solution $F(z, x)$ as well as the transformation kernel $K(x, y)$ associated with $H$ as in (2.17)-(2.21) (replacing | · by an appropriate matrix norm $\|\cdot\|_{\mathbb{C}^{m \times m}}$, have been discussed in great detail in the classical 1963 monograph by Agranovich and Marchenko [1, Ch. I]. Moreover, (2.22)-(2.24) trivially extend to the matrix case.

Given these preliminaries, the analog of Theorem 1.1 and Corollary 2.6 reads as follows in the matrix-valued context.

Theorem 3.1. Let $a>0,0<\varepsilon<\pi / 2$ and suppose

$$
\left\|M_{1}(z)-M_{2}(z)\right\|_{\mathbb{C}^{m \times m}} \underset{|z| \rightarrow \infty}{=} O\left(e^{-2 \operatorname{Im}\left(z^{1 / 2}\right) a}\right)
$$

along the ray $\arg (z)=\pi-\varepsilon$. Then

$$
Q_{1}(x)=Q_{2}(x) \text { for a.e. } x \in[0, a] .
$$

In particular, if (3.13) holds for all $a>0$, then $Q_{1}=Q_{2}$ a.e. on $[0, \infty)$. Sketch of Proof. As in the scalar case, we may assume without loss of generality that

$$
\operatorname{supp}\left(Q_{j}\right) \subseteq[0, a], \quad j=1,2 .
$$

The fundamental identity (2.27), in the present non-commutative case, needs to be replaced by

$$
\frac{d}{d x} W\left(F_{1}(\bar{z}, x)^{*}, F_{2}(z, x)\right)=-F_{1}(\bar{z}, x)^{*}\left[Q_{1}(x)-Q_{2}(x)\right] F_{2}(z, x),
$$

where $F_{j}(z, x)$ denote the $m \times m$ matrix-valued Jost solutions associated with $Q_{j}, j=1,2$, and $W(F, G)(x)=F(x) G^{\prime}(x)-F^{\prime}(x) G(x)$ the matrix-valued Wronskian of $m \times m$ matrices $F$ and $G$. Identity (2.28) then becomes

$$
\begin{aligned}
& \int_{0}^{a} d x F_{1}(\bar{z}, x)^{*}\left[Q_{1}(x)-Q_{2}(x)\right] F_{2}(z, x) \\
& =\left.F_{1}(\bar{z}, x)^{*}\left[M_{1}(z, x)-M_{2}(z, x)\right] F_{2}(z, x)\right|_{x=0} ^{a},
\end{aligned}
$$

utilizing the fact

$$
M_{1}(\bar{z}, x)^{*}=M_{1}(z, x) .
$$


$F_{j}$ obeys a transformation kernel representation

$$
\begin{array}{r}
F_{j}(z, x)=e^{i z^{1 / 2} x} I_{m}+\int_{x}^{a} d y K_{j}(x, y) e^{i z^{1 / 2} y} I_{m}, \\
\operatorname{Im}\left(z^{1 / 2}\right) \geq 0, x \geq 0, j=1,2 .
\end{array}
$$

From this, (3.12), and the hypothesis of (3.13), one concludes by (3.17) that

$$
\int_{0}^{a} d x F_{1}(\bar{z}, x)\left[Q_{1}(x)-Q_{2}(x)\right] F_{2}(z, x) \underset{z \downarrow-\infty}{=} O\left(e^{-2 \operatorname{Im}\left(z^{1 / 2}\right) a}\right) .
$$

Now let $R_{A}$ be right multiplication by $A$ on $n \times n$ matrices and $L_{B}$ be left multiplication by $B$. Then

LHS of (3.20)

$$
=\int_{0}^{a} d x\left\{Q_{1}(y)-Q_{2}(y)+\int_{0}^{y} d x \mathcal{L}(x, y)\left[Q_{1}(x)-Q_{2}(x)\right]\right\} e^{-2 z^{1 / 2} y},
$$

where $\mathcal{L}$ is an operator on $n \times n$ matrices which is a sum of a left multiplication (by $2 K_{j}(x, 2 y-x)$ ), a right multiplication (by $2 K_{2}(x, 2 y-x)$ ), and a convolution of a left and right multiplication.

It follows by Lemma 2.5, (3.20), and (3.21) that

$$
Q_{1}(y)-Q_{2}(y)+\int_{0}^{y} d x \mathcal{L}(x, y)\left[Q_{1}(x)-Q_{2}(x)\right]=0 .
$$

This is a Volterra equation and the same argument based on

$$
\int_{0}^{y} d x_{1} \int_{0}^{x_{1}} d x_{2} \cdots \int_{0}^{x_{n-1}} d x_{n}=\frac{y^{n}}{n !}
$$

that a Volterra operator has zero spectral radius applies to operatorvalued Volterra equations. Thus, (3.22) mplies $Q_{1}(y)-Q_{2}(y)=0$ for a.e. $y \in[0, a]$.

Extensions of Theorem 3.1 in the spirit of Remarks 2.8 2.10 and Theorem 2.11 can be made, but we omit the corresponding details at this point.

Acknowledgments. F. G. thanks T. Tombrello for the hospitality of Caltech where this work was done.

\section{REFERENCES}

[1] Z. S. Agranovich and V.A. Marchenko, The Inverse Problem of Scattering Theory, Gordon and Breach, New York, 1963.

[2] F. V. Atkinson, On the location of the Weyl circles, Proc. Roy. Soc. Edinburgh 88A, 345-356 (1981).

[3] F. V. Atkinson, Asymptotics of the Titchmarsh-Weyl function in the matrix case, unpublished manuscript. 
[4] Ju. M Berezanskii, Expansions in Eigenfunctions of Selfadjoint Operators, Amer. Math. Soc., Providence, RI, 1968.

[5] G. Borg, Uniqueness theorems in the spectral theory of $y^{\prime \prime}+(\lambda-q(x)) y=0$, Proc. 11th Scandinavian Congress of Mathematicians, Johan Grundt Tanums Forlag, Oslo, 1952, pp. 276-287.

[6] R. Carmona and J. Lacroix, Spectral Theory of Random Schrödinger Operators, Birkhäuser, Boston, 1990.

[7] S. Clark and F. Gesztesy, Weyl-Titchmarsh $M$-function asymptotics for matrix-valued Schrödinger operators, preprint, 1999.

[8] W. N. Everitt, On a property of the m-coefficient of a second-order linear differential equation, J. London Math. Soc. 4, 443-457 (1972).

[9] I. M. Gel'fand and B. M. Levitan, On the determination of a differential equation from its special function, Izv. Akad. Nauk SSR. Ser. Mat. 15, 309-360 (1951) (Russian); English transl. in Amer. Math. Soc. Transl. Ser. 2 1, 253$304(1955)$.

[10] F. Gesztesy and B. Simon, Uniqueness theorems in inverse spectral theory for one-dimensinal Schrödinger operators, Trans. Amer. Math. Soc. 348, 349-373 (1996).

[11] F. Gesztesy and B. Simon, A new approach to inverse spectral theory, II. General real potentials and the connection to the spectral measure, preprint, 1998.

[12] F. Gesztesy and B. Simon, in preparation.

[13] D. B. Hinton and J. K. Shaw, On Titchmarsh-Weyl M( $\lambda)$-functions for linear Hamiltonian systems, J. Diff. Eqs. 40, 316-342 (1981).

[14] D. B. Hinton and J. K. Shaw, Hamiltonian systems of limit point or limit circle type with both endpoints singular, J. Diff. Eqs. 50, 444-464 (1983).

[15] D. B. Hinton and J. K. Shaw, On boundary value problems for Hamiltonian systems with two singular points, SIAM J. Math. Anal. 15, 272-286 (1984).

[16] V. I. Kogan and F. S. Rofe-Beketov, On square-integrable solutions of symmetric systems of differential equations of arbitrary order, Proc. Roy. Soc. Edinburgh 74A, 1-40 (1974).

[17] S. Kotani and B. Simon, Stochastic Schrödinger operators and Jacobi matrices on the strip, Commun. Math. Phys. 119, 403-429 (1988).

[18] A. M. Krall, $M(\lambda)$ theory for singular Hamiltonian systems with one singular point, SIAM J. Math. Anal. 20, 664-700 (1989).

[19] A. M. Krall, $M(\lambda)$ theory for singular Hamiltonian systems with two singular points, SIAM J. Math. Anal. 20, 701-715 (1989).

[20] M. G. Krein, Solution of the inverse Sturm-Liouville problem, Doklady Akad. Nauk SSSR 76, 21-24 (1951) (Russian.)

[21] M. G. Krein, On the transfer function of a one-dimensional boundary problem of second order, Doklady Akad. Nauk SSSR 88, 405-408 (1953) (Russian.)

[22] J. Lacroix, The random Schrödinger operator in a strip, in Probability Measures on Groups VII, H. Heyer (ed.), Lecture Notes in Math. 1064, Springer, Berlin, 1984, pp. 280-297.

[23] B. M. Levitan, Inverse Sturm-Liouville Problems, VNU Science Press, Utrecht, 1987.

[24] B. M. Levitan and M. G. Gasymov, Determination of a differential equation by two of its spectra, Russ. Math. Surveys 19:2, 1-63 (1964). 
[25] V. A.Marchenko, Certain problems in the theory of second-order differential operators, Doklady Akad. Nauk SSSR 72, 457-460 (1950) (Russian.)

[26] V. A. Marčenko, Some questions in the theory of one-dimensional linear differential operators of the second order. I, Trudy Moskov. Mat. Obšč. 1, 327-420 (1952) (Russian); English transl. in Amer. Math. Soc. Transl. (2) 101, 1-104 (1973).

[27] V. A. Marchenko, Sturm-Liouville Operators and Applications, Birkhäuser, Basel, 1986.

[28] S. A. Orlov, Nested matrix disks analytically depending on a parameter, and theorems on the invariance of ranks of radii of limiting disks, Math. USSR Izv. 10, 565-613 (1976).

[29] G. Pólya and G. Szegö, Problems and Theorems in Analysis I, Springer, Berlin, 1972.

[30] A. G. Ramm, Property $C$ for ODE and applications to inverse scattering, Z. angew. Analysis 18, 331-348 (1999).

[31] A. G. Ramm, Property $C$ for ODE and applications to inverse problems, preprint, 1999.

[32] F. S. Rofe-Beketov, Selfadjoint extensions of differential operators in a space of vector functions, Sov. Math. Dokl. 10, 188-192 (1969).

[33] B. Simon, A new aproach to inverse spectral theory, I. Fundamental formalism, Annals of Math., to appear.

[34] A. N. Tikhonov, On the uniqueness of the problem of electric prospecting, Doklady Akad. Nauk SSSR 69, 797-800 (1949) (Russian.) 